\title{
Moyamoya disease presenting as Valsalva related partial seizures
}

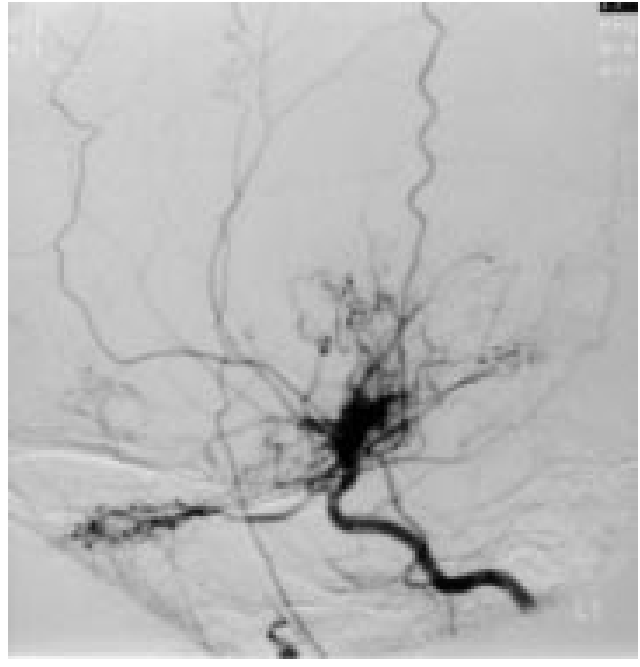

Figure 1 Left internal carotid angiogram showing complete occlusion of the supraclinoid portion of the left internal carotid artery and proximal anterior and middle cerebral artery. Marked collaterals have formed via the deep perforators and leptomeningeal anastomotic channels. These changes were present bilaterally and spared the vertebral-basilar system.

$\mathbf{R}$

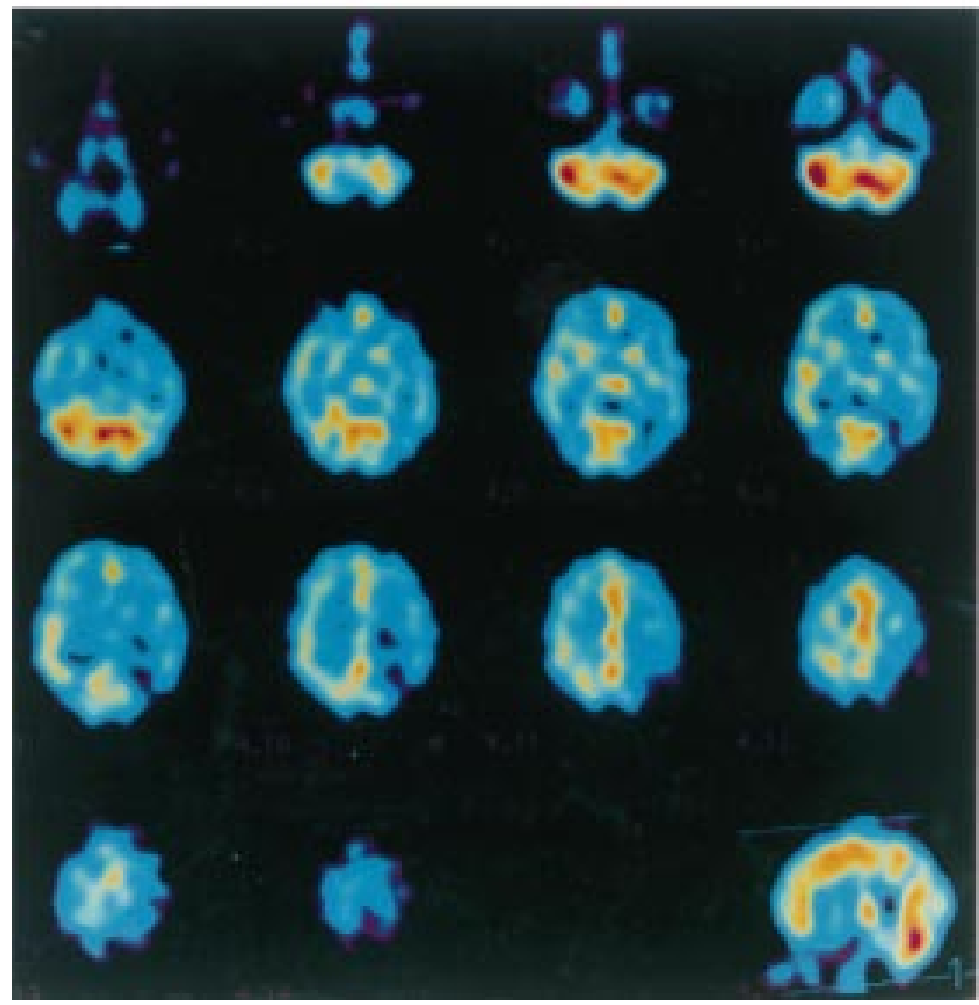

Figure 2 Cerebral perfusion SPECT showing marked reduction in perfusion to the left parietal lobe and patchy perfusion to the frontal lobes bilaterally.
A female patient presented aged 13 with occasional stereotypic episodes of dizziness followed by jerking of her right leg spreading rapidly to her right arm over a few minutes without loss of consciousness. An EEG disclosed a right sided slow wave disturbance which increased with overbreathing and cranial CT was normal. Her seizures resolved on a small dose of carbamazepine. At the age of 19, two years after voluntary cessation of her antiepileptic medication, she was again referred with a brief history of involuntary jerking movements of her right upper limb on straining at stool, occasionally spreading to her leg. These stereotypic episodes would last 1 or 2 minutes and would cease after successful bowel evacuation. Four weeks later she experienced three transient episodes of right limb weakness and a short episode of speech disturbance. One week later she developed worsening, throbbing left frontal headaches.

Cranial CT disclosed a small low density lesion in the left parietal region but was otherwise normal and there was enhancement in a gyral distribution in the left posterior parietal region after gadolinium on MRI suggestive of subacute ischaemia. An EEG showed an excess of delta particularly over the left temporal region. Four vessel angiography showed changes typical of moyamoya disease (fig 1) and a SPECT scan confirmed greatly reduced perfusion to the left parietal region (fig 2).

Moyamoya disease is an idiopathic cerebrovascular disease causing occlusion of the terminal branches of the internal carotid artery and the development of collateral vessels which resemble a puff of smoke on angiography. It has been mainly reported in Japanese children ${ }^{1}$ but increasing numbers of cases are now being reported in non-Japanese adults in whom the most usual presentation is with intracerebral haemorrhage. ${ }^{2}$ Epilepsy is a rarer but recognised presenting feature of moyamoya and this case highlights one of the aetiological mechanisms: The cerebral perfusion pressure can be estimated from the difference between arterial perfusion pressure and intracranial pressure $(\mathrm{CPP}=(\mathrm{AP}-\mathrm{ICP}))$. The Valsalva manoeuvre increases cerebral venous pressure which in turn increases intracranial blood volume and intracranial pressure and reduces arterial perfusion pressure. In areas of cortex already critically perfused this can lead to transient ischaemic damage which may be epileptogenic. It seems likely that the seizure disorder with which she originally presented was also the result of critical perfusion. This patient's symptoms resolved after bilateral extracranial to intracranial synangiosis.

N P ROBERTSON D A S COMPSTON University of Cambridge Neurology Unit

P KIRKPATRICK

University of Cambridge Department of Neurosurgery, Addenbrooke's Hospital, Hills Rd, Cambridge, UK

Correspondence to: Dr N Robertson, University of Cambridge Neurology Unit, Addenbrooke's Hospital, Hills Rd, Cambridge CB2 2QQ, UK. Telephone 01223 216751; fax 01223336941 .

1 Suzuki J, Kodama N. Moyamoya disease-a review. Stroke 1983;14:104-9.

2 Bruno A, Adams HP Jnr, Biller J, et al. Cerebral infarction due to moyamoya disease in young adults. Stroke 1988;19: 826-33. 